\title{
Polar Wander on Triton and Pluto \\ Due to Volatile Migration
}

by

David Parry Rubincam

Geodynamics Branch, Code 921

Laboratory for Terrestrial Physics

NASA's Goddard Space Flight Center

Building 33, Room G308

Greenbelt, MD 20771

voice: 301-614-6464

fax: 301-614-6522

email: rubincam@core2.gsfc.nasa.gov 


\section{Abstract}

Polar wander may occur on Triton and Pluto because of volatile migration. Triton, with its low obliquity, can theoretically sublimate volatiles (mostly nitrogen) at the rate of $\sim 10^{14} \mathrm{~kg} \mathrm{yr}^{-1}$ from the equatorial regions and deposit them at the poles. Assuming Triton to be rigid on the sublimation timescale, after $\sim 10^{5}$ years the polar caps would become large enough to cancel the rotational flattening, with a total mass equivalent to a global layer $\sim 120-250 \mathrm{~m}$ in depth. At this point the pole wanders about the tidal bulge axis, which is the line joining Triton and Neptune. Rotation about the bulge axis might be expected to disturb the leading side/trailing side cratering statistics. Because no such disturbance is observed, it may be that Triton's mantle viscosity is too high but its surface volatile inventory is too low to permit wander. On the other hand, its mantle viscosity might be low, so that any uncompensated cap load might be expected to wander toward the tidal bulge axis. In this case, the axis of wander passes through the equator from the leading side to the trailing side; rotation about this wander axis would not disturb the cratering statistics. Low-viscosity polar wander may explain the bright southern hemisphere: this is the pole which is wandering toward the equator. In any case the "permanent" polar caps may be geologically very young. Polar wander may possibly take place on Pluto, due to its obliquity oscillations and perihelion-pole geometry. However, Pluto is probably not experiencing any wander at present. The Sun has been shining strongly on the poles over the last half of the obliquity cycle, so that volatiles should migrate to the equator, stabilizing the planet against wander. Spacecraft missions to Triton and Pluto which measure the dynamical flattening could give information about the accumulation of volatiles at the poles. Such information is best obtained by measuring gravity and topography from orbiters, as was done for Mars with the highly successful Mars Global Surveyor. 


\section{INTRODUCTION}

Polar wander may occur on Triton and Pluto due to the migration of volatiles. Because of Triton's low obliquity, the Sun shines strongest on the areas near the equator. Insolation falling on Triton's equatorial regions is theoretically strong enough to sublimate the equivalent of a global layer $\sim 120-250 \mathrm{~m}$ thick on a timescale of $\sim 10^{5}$ years and deposit the material at the poles (see Figure 1). This is enough to cancel the flattening due to rotation, so that if the satellite is rigid, Triton overbalances and the pole wanders.

The pole can also wander due to the formation of polar caps if Triton's effective viscosity is low. Consider the Earth. The Earth's pole wanders due to density anomalies caused by tectonics. These anomalies are not nearly large enough to overcome the Earth's equatorial bulge, as they would need to be in the rigid body case. Instead the bulge relaxes fast enough so that it migrates with respect to a coordinate system fixed in the surface; the excess matter associated with the density anomalies eventually ends up at the equator, which is the position of maximum dynamical stability. The mantle relaxation time is about $10^{3}$ years, which is short compared to the $10^{8}$ year tectonic timescale; hence the Earth rapidly adjusts to the tectonic situation, and the pole wanders. It could be the same for Triton and for Pluto, with the wander being caused by the pile-up of volatiles, rather than tectonics The situation for Pluto is more complicated than for Triton because Pluto's obliquity is such that the long-term insolation at the equator is nearly the same as at the poles. If polar wander does occur, it may be associated with the obliquity oscillations and the perihelion-precession geometry. The corresponding figures for Pluto seem likely to be a global layer $\sim 90-180 \mathrm{~m}$ thick sublimated on the obliquity timescale of $\sim 10^{6}$ years. 


\section{TRITON}

Triton orbits Neptune. Triton's orbital plane is tilted with respect to Neptune's equator by inclination $I=156.8^{\circ}$, so that the motion is retrograde. Triton is in synchronous rotation about Neptune, with the satellite's equatorial plane being nearly coincident with its orbital plane. Moreover, Neptune's equator is inclined its own orbital plane about the Sun by $\varepsilon_{N}=29.6^{\circ}$, where $\varepsilon_{\mathrm{N}}$ is Neptune's obliquity. As a result of this geometry, the subsolar point on Triton is always confined to a band between latitudes $\pm 53^{\circ}$ (Harris, 1984; Yelle et $a l ., 1995)$, so that the equatorial regions receive more insolation than the poles. Thus, in addition to seasonal frost deposition (Hansen and Paige, 1992; Spencer and Moore, 1992), there will also be a secular trend to sublimate volatiles (principally nitrogen) in the equatorial regions and condense them at the poles (Brown and Kirk, 1994). This secular trend could have consequences for polar wander, the gravitational field, resurfacing rates, and cratering statistics.

\subsection{Insolation and sublimation on Triton}

Assuming Neptune's orbit about the Sun is circular, the long-term latitudinal second degree $(\ell=2) \Delta F$ component of the insolation incident on Triton can be written as

$$
\Delta F=F_{E}\left(\frac{a_{E}}{a_{N}}\right)^{2}\left(\frac{5}{16}\right)\left(\frac{3}{4} \sin ^{2} \varepsilon_{T}-\frac{1}{2}\right) P_{2}(\sin \phi)
$$

(e.g., Rubincam, 1994), where $F_{\mathrm{E}}=1371 \mathrm{~W} \mathrm{~m}^{-2}$ is the solar constant at the Earth distance of $a_{\mathrm{E}}=1 \mathrm{AU}$ from the Sun, $a_{\mathrm{N}}=30.1 \mathrm{AU}$ is Neptune's distance from the Sun $\mathrm{AU}=$ Astronomical Unit), $\phi$ is latitude on Triton, $\varepsilon_{\mathrm{T}}$ is Triton's obliquity relative to the Sun, and 


$$
P_{2}(\sin \phi)=\left(3 \sin ^{2} \phi-1\right) / 2
$$

is the second degree Legendre polynomial. The obliquity $\varepsilon_{\mathrm{T}}$ constantly changes because Triton's orbit precesses on Neptune's equatorial plane with a period of 600 to 700 years (e.g., Harris, 1984; Jacobson, 1990). The relationship is

$$
\cos \varepsilon_{T}=\cos I \cos \varepsilon_{N}+\sin I \sin \varepsilon_{N} \cos \Omega^{\prime}\left(t-t_{0}\right)
$$

where $\Omega^{\prime}$ is the precession angular speed, $t$ is time, and $t_{0}$ is some reference time. Plugging this equation into (1) and averaging over time yields

$$
<\Delta F>=F_{\mathrm{E}}\left(a_{E} / a_{\mathrm{N}}\right)^{2}\left[(5 / 16) P_{2}(\sin \phi)\left(2-6 \cos ^{2} I \cos ^{2} \varepsilon_{\mathrm{T}}-3 \sin ^{2} I \sin ^{2} \varepsilon_{\mathrm{T}}\right) / 8\right]
$$

which, for the values of $I$ and $\varepsilon_{\mathrm{N}}$ given above, yields

$$
<\Delta F>=-0.125 P_{2}(\sin \phi) \mathrm{W} \mathrm{m} \mathrm{m}^{-2}
$$

so that there is excess insolation in the equatorial regions, as stated above. For a typical area of $2 \pi R_{\mathrm{T}}{ }^{2}$ (where $R_{\mathrm{T}}=1352 \mathrm{~km}$ is the radius of Triton), an insolation value of $\sim 0.125 \mathrm{~W} \mathrm{~m}$ 2, an albedo $A \cong 0.8$, and a latent heat of sublimation of $2.5 \times 10^{5} \mathrm{~J} \mathrm{~kg}^{-1}$ (Spencer et al., 1997, p. 454), the rate of sublimation $S$ is approximately

$$
(1-0.8)(2)(3.14)\left(1.352 \times 10^{6} \mathrm{~m}\right)^{2}\left(0.125 \mathrm{~W} \mathrm{~m}^{-2}\right)\left(3.16 \times 10^{7} \mathrm{~s} \mathrm{yr}^{-1}\right) /\left(2.5 \times 10^{5} \mathrm{~J} \mathrm{~kg}^{-1}\right) \text {, }
$$

or an amount 


$$
S \cong 3.6 \times 10^{13} \mathrm{~kg} \mathrm{yr}^{-1}
$$

can be sublimated from the equatorial regions for redeposition at the poles. This is much greater than the $\sim 10^{9}-10^{10} \mathrm{~kg} \mathrm{yr}^{-1}$ produced by the plumes observed so far on Triton (Kirk et al., 1995). Also, (5) works out to be approximately $36 \mathrm{~km}^{3} \mathrm{yr}^{-1}$, which is much greater than the resurfacing rate conservatively estimated at $0.01 \mathrm{~km} \mathrm{yr}^{-1}$ by Stern and McKinnon (2000). Hence the rate of volatile movement can theoretically be quite high.

\subsection{Mass distribution and gravitational field}

The next step is to find how this sublimation might affect the mass distribution on Triton. The mass distribution is most conveniently expressed in terms of Triton's gravitational field, which can be written in the usual spherical harmonic form

$$
V(r, \phi, \lambda)=\frac{G M_{T}}{r} \sum_{\ell=0}^{\infty}\left(\frac{R_{T}}{r}\right)^{\ell} \sum_{m=0}^{\ell} P_{\ell m}(\sin \phi)\left(C_{\ell m} \cos m \lambda+S_{\ell m} \sin m \lambda\right)
$$

(e.g., Kaula, 1966; Caputo, 1967), where $V(r, \phi, \lambda)$ is the gravitational potential, $r$ is the radial distance from the center of mass, $\phi$ is latitude, $\lambda$ is east longitude, and $G=6.673 \times 10^{-11} \mathrm{~m}^{3}$ $\mathrm{kg}^{-1} \mathrm{~s}^{-2}$ is the universal constant of gravitation, while $M_{\mathrm{T}}=2.14 \times 10^{22} \mathrm{~kg}$ is the mass of Triton. The $P_{\ell \mathrm{m}}(\sin \phi)$ are the associated Legendre polynomials, while the $C_{\ell \mathrm{m}}$ and $S_{\ell \mathrm{m}}$ are unnormalized spherical harmonic coefficients. The convention among geodesists is to speak of the zonal $C_{\ell 0}$ coefficients in terms of $J_{\ell}$, where simply $J_{\ell}=-C_{\ell 0}$.

Triton feels centrifugal forces from rotation, with the rotational potential being 


$$
V_{r o t}=\left(\frac{\omega_{P}^{2} r^{2}}{3}\right)\left[P_{2}(\sin \phi)+2\right]
$$

where $P_{2}(\sin \phi)=P_{20}(\sin \phi)$. This potential tends to flatten the satellite, making Triton somewhat pumpkin-shaped. Triton's responds to this potential with $J_{2, \text { rot }}$, the second degree spherical harmonic coefficient due to the rotational hydrostatic flattening (e.g., Kaula, 1968, pp. 68-73):

$$
J_{2, \text { rot }} \cong \frac{\omega_{T}^{2} R_{T}^{3}}{3 G M_{T}}\left[\frac{5}{1+\left(\frac{5}{2}-\frac{15}{4} \lambda_{T}\right)^{2}}-1\right]
$$

In the above equation $\omega_{\mathrm{T}}=2 \pi /(5.877$ days $)$ is the angular speed of 'Triton's rotation about its axis, while

$$
\lambda_{\mathrm{T}}=C_{\mathrm{T}} / M_{\mathrm{T}} R_{\mathrm{T}}^{2}
$$

is the central condensation factor, with $C_{\mathrm{T}}$ being its polar moment of inertia. Here $\lambda_{\mathrm{T}}$ measures how much differentiation has taken place; it should not be confused with the $\lambda$ which is used for longtiude in (6). At present $C_{\mathrm{T}}$ is unknown, but substituting $\lambda_{\mathrm{T}}=0.4$, the value for a homogeneous sphere, yields by (8) a maximum value for $J_{2, \text { rot }}$ of

$$
J_{2, \text { rot }} \approx 1.325 \times 10^{-4}, \quad \lambda_{\mathrm{r}}=0.4 .
$$


Rotation is not the only contributor to the gravitational field; the bulge raised by Neptune's tidal attraction will also give rise to $C_{\ell \mathrm{m}}$ and $S_{\ell \mathrm{m}}$ coefficients. Neptune's tidal potential is

$$
V_{\text {tide }}=\frac{G M_{N} r^{2}}{a^{3}} P_{2}(\cos \Psi)
$$

where $\Psi$ is the angle measured from the line joining Triton and Neptune, $M_{\mathrm{N}}=1.024 \times 10^{26}$ $\mathrm{kg}$ is Neptune's mass, and $a=3.548 \times 10^{8} \mathrm{~m}$ is Triton's distance from Neptune. The tidal potential tends to stretch Triton into a shape something like an American football, with the long end pointing to Neptune. It is interesting to note that the ratio of the coefficients of the Legendre polynomial in (7) and (11) is (tides/rotation) $=3 G M_{\mathrm{N}} / \omega_{\mathrm{T}}^{2} a^{3} \approx 3$. The tidal potential is thus 3 times stronger than the rotational potential, so that most of Triton's flattening is due to tides and not rotation. In other words, Triton has a distinctly triaxial shape, being more of a football than a pumpkin, with the axis of the football in the equatorial plane.

The $C_{\ell \mathrm{m}}$ and $S_{\ell \mathrm{m}}$ tidal coefficients can be found with the help of the addition theorem (e.g., Kaula, 1968, p. 67):

$$
P_{\ell}(\cos \Psi)=\sum_{m=0}^{\ell} \frac{\left(2-\delta_{0 m}\right)(\ell-m) !}{(\ell+m) !} P_{\ell m}\left(\sin \phi_{N}\right) P_{\ell m}(\sin \phi) \cos \left[m\left(\lambda-\lambda_{N}\right)\right]
$$

Here $\delta_{\mathrm{ij}}$ is the Kronecker delta, defined such that $\delta_{\mathrm{ij}}=1$ for $i=j$ and is 0 otherwise. In the above equation $\phi_{\mathrm{N}}$ and $\lambda_{\mathrm{N}}$ are the respective latitude and east longitude of the sub-Neptune point. Apart from libration, Neptune will be on Triton's equator, and by convention longitude is measured from the sub-Neptune point. Libration will be ignored, so that $\phi_{\mathrm{N}}=0$ 
and $\lambda_{\mathrm{N}}=0$ for the purposes considered here. Thus substituting (12) into (11) yields by analogy with (8)

$$
J_{2, \text { tide }} \cong \frac{R_{T}^{3} M_{N}}{2 a^{3} M_{T}}\left[\frac{5}{1+\left(\frac{5}{2}-\frac{15}{4} \lambda_{T}\right)^{2}}-1\right]
$$

for the equilibrium tides, which gives

$$
J_{2, \text { ide }}=1.986 \times 10^{-4}, \quad \lambda_{\mathrm{T}}=0.4
$$

for the $J_{2}$ component of the tidal bulge raised on a homogeneous Triton by Neptune. The flattening from the tidal potential is thus larger than the rotational flattening. The total hydrostatic coefficient will be

$$
J_{2, \text { hydro }}=J_{2, \text { rot }}+J_{2, \text { ide }} \approx 3.311 \times 10^{-4}, \quad \lambda_{\mathrm{T}}=0.4
$$

It may be that $J_{2, \text { rot }}$ is smaller than given by (15) because Triton is differentiated, as would be expected if it was captured into orbit by slamming into previously existing moons and was greatly heated by that and the tidal circularization of its orbit (Goldreich et al., 1989). If Triton is highly differentiated, as seems likely from the collision scenario, then if the rocky core has a constant density $\rho_{\mathrm{r}}$ and the icy mantle a constant density $\rho_{\mathrm{i}}$, the condensation factor (9) can be shown to be (Rubincam, 2000)

$$
\lambda_{T}=\frac{C_{T}}{M_{T} R_{T}^{2}}=\frac{2}{5}\left[\frac{\left(\rho_{T}-\rho_{i}\right)^{5 / 3}}{\rho_{T}\left(\rho_{r}-\rho_{i}\right)^{2 / 3}}+\frac{\rho_{i}}{\rho_{T}}\right]
$$


where $\rho_{\mathrm{T}}=2064 \mathrm{~kg} \mathrm{~m}^{-3}$ is Triton's mean density (e.g., McKinnon et al., 1995). Water ice with density $\rho_{\mathrm{i}}=1000 \mathrm{~kg} \mathrm{~m}^{-3}$ and anhydrous rock with density $\rho_{\mathrm{r}}=3500 \mathrm{~kg} \mathrm{~m}^{-3}$ give $\lambda_{\mathrm{T}}=$ 0.311. On the other hand, for hydrated rock with density $\rho_{\mathrm{r}}=2800 \mathrm{~kg} \mathrm{~m}^{-3}$ (McKinnon et al., 1997, p. 302), $\lambda_{\mathrm{T}}$ could only get as low as 0.339 . Assuming a worst case value of $\lambda_{\mathrm{T}} \approx$ 0.300 gives

$$
\begin{array}{ll}
J_{2, \text { rot }} \approx 0.645 \times 10^{-4}, & \lambda_{\mathrm{T}} \approx 0.3 \\
J_{2, \text { tide }} \approx 0.966 \times 10^{-4}, & \lambda_{\mathrm{T}} \approx 0.3
\end{array}
$$

and

$$
J_{2, \text { hydro }}=J_{2, \text { rot }}+J_{2, \text { ide }} \approx 1.611 \times 10^{-4}, \quad \lambda_{\mathrm{T}} \approx 0.3 .
$$

For principal axis rotation $C_{21}=S_{21}=0$. Also, $S_{22}=0$ by (12) because $\lambda_{\mathrm{N}}=0$. Thus the only other second degree coefficient will be $C_{22}$, given by

$$
C_{22, \text { ide }} \cong \frac{R_{T}^{3} M_{N}}{4 a^{3} M_{T}}\left[\frac{5}{1+\left(\frac{5}{2}-\frac{15}{4} \lambda_{T}\right)^{2}}-1\right]
$$

which gives

$$
C_{22, \text { ide }}=0.993 \times 10^{-4}, \quad \lambda_{\mathrm{T}}=0.4
$$

for a homogeneous Triton, and 


$$
C_{22, \text { ide }}=0.483 \times 10^{-4}, \quad \lambda_{\mathrm{T}}=0.3
$$

for a highly differentiated Triton. The numbers computed here are summarized in Table 1.

\subsection{Rigid body (high interior viscosity) case}

Uncompensated polar volatile deposits will act to decrease $J_{2}$ because they add mass to the poles, making the satellite dynamically rounder. This section calculates the time necessary for a rigid Triton to overcome the rotational flattening. A more rigorous but less physically obvious derivation is relegated to the Appendix.

Assuming that the ice deposit has the shape of a spherical cap centered on the pole with a mass $M_{\text {cap }}$, angular extent $\psi$, and has a uniform thickness, the contribution to $J_{2}$ will be

$$
J_{2, \text { cap }}=-M_{\text {cap }}\left(\cos \psi+\cos ^{2} \psi\right) /\left(2 M_{\mathrm{T}}\right)
$$

(Rubincam, 1992, p. 2630). Assuming a somewhat arbitrary angular size of $\psi \cong 45^{\circ}$ makes the above expression become

$$
J_{2, \text { cap }} \approx-M_{\text {cap }} /\left(2 M_{\mathrm{T}}\right)
$$

with smaller cap sizes giving bigger contributions to $J_{2}$.

If the effective viscosity of Triton's mantle is high, so that Triton can be considered rigid on the sublimation timescale, then the polar deposits must overcome the rotational flattening for polar wander to occur. This happens when 


$$
J_{2, \text { rot }}+J_{2, \text { cap }}=0 \text {. }
$$

The polar caps cancel the rotational flattening and dynamically only the tidal bulge is left; Triton is then free to rotate about the Neptune-Triton line, i.e., the pole is free to wander about the axis of the tidal bulge, as shown in Figure 2. Any uncompensated mass centered on the pole will be in unstable equilibrium, so that any perturbation will cause Triton to rotate to try and place the excess mass on the equator. Thus the time $t_{\mathrm{S}}$ required for sublimation to overcome the rotational flattening will, by (5), (10), (22), and (23), be given by

$$
t_{\mathrm{S}} \cong M_{\text {cap }} / S=2 M_{\mathrm{T}} J_{2, \text { rot }} / \mathrm{S} \approx 1.6 \times 10^{5} \mathrm{yr}, \quad \lambda_{\mathrm{T}}=0.4
$$

for a homogeneous Triton, where the amount of volatiles involved is

$$
M_{\text {cap }} \approx 6 \times 10^{18} \mathrm{~kg}, \quad \lambda_{\mathrm{T}}=0.4
$$

which is equivalent to a global layer $250 \mathrm{~m}$ deep, assuming a density of $1000 \mathrm{~kg} \mathrm{~m}^{-3}$. For a highly differentiated Triton the numbers are

$$
t_{\mathrm{S}} \cong M_{\text {cap }} / S=2 M_{\mathrm{T}} J_{2, \text { rot }} / \mathrm{S} \approx 0.8 \times 10^{5} \mathrm{yr}, \quad \lambda_{\mathrm{T}}=0.3
$$

and

$$
M_{\text {cap }} \approx 3 \times 10^{18} \mathrm{~kg}, \quad \lambda_{\mathrm{T}}=0.3 .
$$


The above figures are the equivalent of a global layer only $120 \mathrm{~m}$ deep and a timescale of just $\sim 8 \times 10^{4}$ yr. On the other hand, there may be some nonhydrostatic (e.g., tectonic or impact) component of unknown size to overcome, so that $t_{\mathrm{S}}$ and $M_{\text {cap }}$ might be larger than given above. These results are summarized in Table 1.

\subsection{Low interior viscosity case}

The above values for $t_{\mathrm{S}}$ and $M_{\text {cap }}$ assumed Triton to be a rigid body. In reality, the surface will relax under the load and $J_{2, \text { cap }}$ will decay according to a characteristic time. For a homogeneous viscous planet the $\ell=2$ Darwin relaxation time $t_{\mathrm{D}}$ toward isostatic compensation is (Darwin, 1879):

$$
t_{\mathrm{D}}=19 \eta_{\mathrm{T}} /\left(2 \rho_{\mathrm{T}} g_{\mathrm{T}} R_{\mathrm{T}}\right)
$$

where $\eta_{\mathrm{T}}$ is Triton's effective viscosity, $\rho_{\mathrm{T}}=2064 \mathrm{~kg} \mathrm{~m}^{-3}$ is Triton's mean density, and $g_{\mathrm{T}}=$ $0.78 \mathrm{~m} \mathrm{~s}^{-2}$ is the surface gravitational acceleration (e.g., McKinnon et al., 1995). The viscosity $\eta_{\mathrm{T}}$ is not known. For comparison, an Earth-like mantle viscosity of $\eta_{\mathrm{T}}=10^{21} \mathrm{~Pa} \mathrm{~s}$ yields

$$
t_{\mathrm{D}}=1.38 \times 10^{5} \mathrm{yr}, \quad \quad \eta_{\mathrm{T}}=10^{21} \mathrm{~Pa} \mathrm{~s}
$$

and the relaxation time is on the order of the sublimation time $t_{\mathrm{s}}$.

Isostatically compensated mass anomalies "float" on the substrate like icebergs and have only a small effect on the gravity field and moments of inertia. Thus the sinking of the ice load toward isostacy inhibits polar wander. On the other hand, a low interior viscosity means that the rotational and tidal bulges can relax quickly, so that the pole will wander 
southward in order to place whatever uncompensated ice load there is on the equator. This situation is more complicated than the rigid body case and will not be treated in detail here, except to make two remarks.

The first remark is that in the low viscosity case the ice load need not overcome the rotational flattening for the pole to wander, as it did in the rigid body case, so that the uncompensated ice cap need not be as thick. This is analogous to the situation on the Earth: plate tectonics causes the pole to wander, even though the mass associated with tectonics is nowhere near large enough to overcome the Earth's huge rotational flattening. On the Earth values of $C_{2 \mathrm{~m}}$ and $S_{2 \mathrm{~m}}$ having typical magnitudes of $10^{-6}$, while $J_{2} \cong 10^{-3}$. But the Earth's rotational bulge relaxes fast enough to allow the pole to shift, thanks to a mantle viscosity which gives a $t_{\mathrm{D}}=\sim 10^{3} \mathrm{yrs}$, which is short relative to the $10^{8} \mathrm{yr}$ tectonic timescale (e.g., Stacey, 1992, pp. 394-400; Lambeck, 1980, pp. 342-346). Likewise, ice ages on the Earth create ice caps which cause a certain amount of polar wander, even though these caps are nowhere near the size of the equatorial bulge (e.g., Bills and James, 1997).

The second remark is that in the low viscosity case, Triton's pole might be expected to wander in the direction shown in Figure 3, so that Triton attempts to place the extra mass not just on the equator, but on the axis of the tidal bulge, assuming that there are no other nonhydrostatic loads. The reason for putting it there is that this is the position of maximum dynamical stability, just as the extra mass on the Moon's equator is at the sub-Earth subanti-Earth axis. The sense of rotation is $90^{\circ}$ away from the rigid body case. If there are such nonhydrostatic loads, as might be created by convection in Triton's interior (analogous to what happens on Earth), then it is not clear what the polar wander path is. It might be a random walk, as it is on Earth; or if the ice load overwhelms the other sources, it may still be predominately as shown in Figure 3. Low-viscosity polar wander may be the reason for the large observed south polar cap, as discussed later (section 4). 


\section{PLUTO}

Pluto can be treated in a way analogous to Triton. As with Triton, the migration of volatiles to Pluto's poles can have consequences for resurfacing rates, polar wander, the gravitational field, and cratering statistics.

\subsection{Pluto insolation}

For Pluto the expression analogous to (1) is

$$
<\Delta F>=F_{E}\left(\frac{a_{E}}{a_{P}}\right)^{2}\left(\frac{5}{16}\right)\left(\frac{3}{4} \sin ^{2} \varepsilon_{P}-\frac{1}{2}\right) P_{2}(\sin \phi)
$$

where $a_{\mathrm{P}}=40 \mathrm{AU}$ is Pluto's distance from the Sun and $\varepsilon_{\mathrm{p}}$ is Pluto's obliquity. Pluto's obliquity oscillates with time according to the equation

$$
\varepsilon_{\mathrm{p}}=115.5^{\circ}+11.8^{\circ} \sin \chi
$$

where $\chi=19.5^{\circ}+130.2^{\circ} t$, with time $t$ being measured in millions of years from the present time (Dobrovolskis and Harris, 1983; Dobrovolskis et al., 1997). Thus Pluto's obliquity oscillates between $\sim 103.7^{\circ}$ and $\sim 127.3^{\circ}$ with a $2.8 \times 10^{6}$ period. For obliquities less than $\sim 125^{\circ}$ the poles receive more insolation than the equator; see Ward (1974) and (30) above. Therefore Pluto spends nearly all of its time with the poles receiving more sunlight than the equator (e.g., Dobrovolskis et al., 1997; Spencer et al., 1997).

A naive interpretation of this situation would be that over the long term, volatiles flee to the equator, making the planet if anything flatter instead of rounder, so that there is no 
polar wander on Pluto. However, it may be that Pluto's climate dynamics do not decree that volatile migration goes to zero at the obliquity where the polar insolation exactly equals the equatorial insolation (Rubincam, 2000). Perhaps instead of occuring at $\varepsilon_{\mathrm{p}} \approx 125^{\circ}$; volatile migration reverses course nearer its present average obliquity of $115.5^{\circ}$. It might even be that Pluto has altered its average obliquity through the phenomenon of climate friction so that it is now in a state of no net transport of volatiles over geologic time; however, it is by no means clear at present whether climate friction can have either the magnitude or the direction required to perform this feat (Rubincam, 2000).

If Pluto's average obliquity is near a state of zero net transport, then volatiles may periodically shuttle into and out of the polar regions as the obliquity oscillates. Volatile movement may also depend on the perihelion-spin pole geometry. Pluto's orbit about the Sun is highly eccentric, with an orbital eccentricity of 0.25 . Pluto's spin pole precesses on its orbital plane with a period of $2.8 \times 10^{6}$ years (Dobrovolskis et al., 1997). As Pluto precesses, it exposes first its equator and then the poles to the most sunlight at perihelion. The question becomes whether during times of polar deposition volatiles accumulate to the point where the pole wanders.

In the absence of a detailed climate model, a typical insolation deficit will be assumed to be a factor of 10 lower than for Triton:

$$
\Delta F=-0.01 P_{2}(\sin \phi) \mathrm{W} \mathrm{m} \mathrm{m}^{-2}
$$

giving, in a calculation analogous to (4),

$$
S=2.1 \times 10^{12} \mathrm{~kg} \mathrm{yr}^{-1}
$$

using $R_{\mathrm{P}}=1150 \mathrm{~km}$ as the radius of Pluto. 


\subsection{Rigid body (high viscosity case)}

The rotational and tidal flattenings can be computed as was done for Triton. Just as Neptune raises a tidal bulge on Triton, so Charon raises a tidal bulge on Pluto. Using the values $M_{\mathrm{P}}=1.288 \times 10^{22} \mathrm{~kg}, M_{\mathrm{C}}=1.891 \times 10^{21} \mathrm{~kg}, \omega_{\mathrm{P}}=2 \pi /(6.387$ days $)$, and $a=19,662$ $\mathrm{km}$ in (8) and (13) yields

$$
J_{2, \text { rot }}=1.146 \times 10^{-4}, \quad \lambda_{P}=0.4
$$

and

$$
J_{2, \text { tide }}=0.220 \times 10^{-4}, \quad \lambda_{\mathrm{P}}=0.4
$$

leading to

$$
J_{2, \text { hydro }}=J_{2, \text { rot }}+J_{2, \text { ide }}=1.366 \times 10^{-4}, \quad \lambda_{\mathrm{p}}=0.4
$$

It will be seen that the hydrostatic tidal flattening relative to rotational flattening is much smaller for Pluto than for Triton, but is still significant. These numbers lead to

$$
t_{\mathrm{S}}=2 M_{\mathrm{P}} J_{2, \text { rot }} / \mathrm{S} \approx 1.6 \times 10^{6} \mathrm{yr}, \quad \lambda_{\mathrm{P}}=0.4
$$

by analogy to (16), and giving

$$
M_{\text {cap }}=3 \times 10^{18} \mathrm{~kg}, \quad \lambda_{\mathrm{P}}=0.4
$$


for a rigid Pluto. This is equivalent to a global layer $180 \mathrm{~m}$ deep. Since $t_{\mathrm{S}}$ is about half the obliquity oscillation period and the precession period, polar wander seems barely possible if Pluto is rigid, assuming the supply of volatiles is great enough for the planet to overbalance. For a highly differentiated Pluto, the corresponding figures are

$$
J_{2, \text { rot }}=0.558 \times 10^{-4}, \quad \lambda_{\mathrm{P}}=0.3
$$

and

$$
J_{2, \text { ide }}=0.107 \times 10^{-4}, \quad \lambda_{\mathrm{P}}=0.3
$$

leading to

$$
\begin{array}{cc}
J_{2, \text { hydro }}=0.665 \times 10^{-4}, & \lambda_{\mathrm{P}}=0.3 \\
t_{\mathrm{S}}=2 M_{\mathrm{P}} J_{2, \text { rot }} / \mathrm{S} \approx 8 \times 10^{5} \mathrm{yr}, & \lambda_{\mathrm{P}}=0.3
\end{array}
$$

and giving

$$
M_{\text {cap }}=2 \times 10^{18} \mathrm{~kg}, \quad \quad \lambda_{\mathrm{p}}=0.3 .
$$

This last value is equivalent to global layer whose thickness is $85 \mathrm{~m}$.

The $C_{22, \text { tide }}$ term can be calculated for Pluto by using the analog of (18). The result is 


$$
\begin{array}{cc}
C_{22, \text { tide }}=0.110 & \lambda_{\mathrm{P}}=0.4 \\
C_{22, \text { ide }}=0.054 & \lambda_{\mathrm{P}}=0.3 .
\end{array}
$$

These results are summarized in Table 1.

\subsection{Low viscosity case}

For Pluto $\rho_{\mathrm{P}}=2022 \mathrm{~kg} \mathrm{~m}^{-3}, g_{\mathrm{P}}=0.65 \mathrm{~m} \mathrm{~s}^{-2}$, so that the Plutonian equivalent of (28) is

$$
t_{\mathrm{D}}=19 \eta_{\mathrm{P}} /\left(2 \rho_{\mathrm{P}} g_{\mathrm{P}} R_{\mathrm{P}}\right)
$$

This gives

$$
t_{\mathrm{D}} \approx 2 \times 10^{5} \mathrm{yr}, \quad \quad \eta_{\mathrm{P}}=10^{21} \mathrm{Pas}
$$

where $10^{21} \mathrm{~Pa}$ is an Earth-like mantle viscosity. Unlike the case for Triton, $t_{\mathrm{D}}$ is shorter than the assumed value for $t_{\mathrm{S}}$ if Pluto has an Earth-like viscosity. Marcialis (1985) has argued that Pluto in fact has a very low interior viscosity, so that the planet relaxes very quickly, yielding a topographically bland planet.

The direction of Pluto's polar wander will be analogous to that of Triton. In the rigid body case it will be as shown in Figure 2; while for a low viscosity it will presumably be as shown in Figure 3, assuming no other nonhydrostatic loads. 


\section{DISCUSSION}

For an Earth-like mantle viscosity of $10^{21} \mathrm{~Pa} \mathrm{~s}$, Triton's relaxation time $t_{\mathrm{D}}$ is about the same as the sublimation time $t_{\mathrm{S}}$; while for Pluto $t_{\mathrm{D}}$ is shorter than $t_{\mathrm{S}}$. The actual mantle viscosities of both Triton and Pluto are unknown. McKinnon et al. (1997, p. 316) use a viscosity estimate of $\eta=139 \exp (A / T)$ Pa s for Pluto's water ice mantle, where $A=6830 \mathrm{~K}$ and $T$ is measured in kelvins. This equation gives $\eta>10^{21} \mathrm{~Pa}$ s for the outer $\sim 150 \mathrm{~km}$ of the planet for a particular thermal model, but obviously the viscosity is very sensitive to the chosen value of $A$.

Triton's total planetary inventory of nitrogen has been estimated at a global surface layer equivalent of $\sim 1 \mathrm{~km}$ (Brown and Kirk, 1994; Yelle et al., 1995, p. 1068). This is close to the $\sim 120-250 \mathrm{~m}$ necessary for rigid body wander to occur, so that a substantial fraction of all its nitrogen would have to be on the surface. Also, Triton's cratering statistics argue against rigid body wander having occurred over the age of the current surface. Triton's leading side (apex of motion) has more craters than its trailing side (Croft et al., 1995). If Triton's interior viscosity is high compared to the sublimation timescale, then the pole would be expected to wander in the sense shown in Figure 2, which would tend to smear out the leading side/trailing side asymmetry and produce a symmetrical ring whose axis is the Triton-Neptune line, assuming the pole wanders continuously in one direction. Hence the current data seem consistent with Triton having a high mantle viscosity but with a low surface volatile supply, the supply being too small to overcome the flattening. However, it may be that Triton rocks back and forth about the bulge axis instead of rolling continuously; in such a case the smearing would be less.

On the other hand, the cratering statistics may be compatible with polar wander taking place on a low viscosity interior. In this situation, the pole would be expected to wander in the sense shown in Figure 3 if the caps are the only uncompensated loads, so that the axis of wander pierces the leading side and trailing side on the equator. Rotation about 
this axis will not disturb the leading side/trailing side asymmetry. Another argument for a low viscosity is that Triton could be very active geologically (Stern and McKinnon, 2000), which may mean that the interior is hot and its viscosity relatively low. Certainly if Triton has a subsurface ocean the effective viscosity will be extremely low.

It must be stated that only $40 \%$ of Triton's surface has been seen clearly enough to yield useful cratering statistics (Stern and McKinnon, 2000). The other half of the satellite may yield something interesting.

If polar wander does occur, then ice in the caps is brought closer to the equator, where sunlight can once again sublime the material for transport back to the poles. On Triton deposition is expected to be more or less continuous, so that the pole will always be wandering. This will have implications for the thickness of the ice caps and for resurfacing the satellite.

If Triton can be considered rigid on the sublimation timescale, then for two ice caps each with angular size of $45^{\circ}$, the ice will cover the topography to an average depth no greater than $\sim 850 \mathrm{~m}$. The corresponding number for Pluto is $\sim 615 \mathrm{~m}$. If the mantle viscosity of either body is low, then presumably the depths are even smaller. In any case there will be a limit to the depths of the ice caps on the order of hundreds of meters.

Brown and Kirk (1994) propose the viscous spreading of the caps to explain why Triton has no thick ice caps concentrated at the poles, but polar wander seems a more likely explanation: the deposits move south and are sublimated before they can form thick sheets. The speed at which the pole wanders is not computed here, but the timescale would be expected to be short, so that the "permanent" polar caps would be geologically very young.

Polar wander may be the explanation for the anomalously bright southern hemisphere: perhaps it is this hemisphere which is wandering toward the equator, bringing the bright surface deposits with it, while the northern hemisphere tilts back out of view. Another possibility is that the bright feature is a seasonal polar cap (Yelle et al., 1995).

("North" and "south" here follow the convention of referring to these directions 
on Neptune and not Triton. But under the normal assumption that the positive spin vector pierces the northern hemisphere, the bright hemisphere is actually Triton's northern hemisphere. Recall Triton's motion is retrograde.)

A spacecraft mission to Triton might give information about the moon's stiffness and the migration of volatiles. Suppose polar wander occurs continuously on Triton; then for a rigid interior, the moment of inertia about its rotation axis would always be near the limit of stability, and Triton's gravitational field would be dramatically different from what is expected at hydrostatic equilibrium due to rotation and tides. For instance, if the spacecraft measured a $J_{2}$ much lower than the $J_{2, \text { hydro }}$ given by (17) for a highly differentiated Triton, then this would be dramatic evidence for polar wander.

A spacecraft measurement of Pluto's $J_{2}$ would be less clear-cut. Pluto's obliquity has just passed through a period of "low" obliquity, "low" in this case meaning values closer to the minimum numerical value of $104^{\circ}$ rather than the high of $127^{\circ}$, as can be seen from (31). Maximum insolation on the poles occurs at $90^{\circ}$ obliquity and decreases at higher values. So the Sun has been shining as much as it ever does on Pluto's poles for the last $\sim 1.5 \times 10^{6}$ years or so. Hence volatiles might be expected to sublimate and the poles accumluate at the equator, stabilizing the planet against wander and leading to a greater than average value of $J_{2}$ at the present time. But excess mass from any other source, such as tectonic activity, would be expected to end up at the equator and increase $J_{2}$, just as is the case on Earth. Hence there would be a problem in determining from spacecraft how much of the excess mass is due to volatile migration and how is much due to other sources.

Whether polar wander occurs on Pluto due to volatile migration is problematic. For the simple climate model given by (30), volatile accumulation at the poles takes place only when the obliquity is near its extreme value of $127.3^{\circ}$. The value for $S$ given by (33) is an extremely crude estimate, and assumes (30) is not quite the right model. A better climate model is needed for Pluto to see whether the wander is actually possible. 
Should one or the other of the gravitational fields be unexpectedly quiet, with only hydrostatic components present indicative of a fluid body, then the moment of inertia could be found from inverting (18) as well as the sum of (8) and (13). This yields

$$
\lambda_{T}=\frac{2}{3}-\frac{4}{15} \sqrt{\frac{5}{\frac{6 J_{2, \text { tot }}}{G \omega_{T}^{2} R_{T}^{3}}+3\left(\frac{M_{N}}{M_{T}}\right)\left(\frac{R_{T}}{a}\right)^{3}}+1}-1
$$

using the $J_{2, \text { tot }}$ coefficient and

$$
\lambda_{T}=\frac{2}{3}-\frac{4}{15} \sqrt{\frac{5}{4\left(\frac{M_{T}}{M_{N}}\right)\left(\frac{a}{R_{T}}\right)^{3} C_{22, \text { tide }}+1}-1}
$$

using the $C_{22, \text { tide }}$ coefficient for Triton, with corresponding equations for Pluto, giving estimates on the degree of differentiation inside Triton and/or Pluto. For instance, for the Earth the observed value for $J_{2}$ is $1.0826 \times 10^{-3}$ (e.g., Lerch et al., 1979; Stacey, 1992, p. 136), which by (45) leads to $\lambda_{E}=0.332$, a figure which is basically indistinguishable from that obtained from precession (e.g., Stacey, 1992, p. 33). The reason the numbers are so close is that the Earth's rotational flattening dwarfs all other sources of the gravitational field. On the other hand, for Mars $J_{2}=1.959 \times 10^{-3}$ (Smith et al., 1993), yielding $\lambda_{\mathrm{M}}=$ 0.375. The observed value from precession is $\lambda_{\mathrm{M}}=0.365$ (Folkner et al., 1997); which is smaller than the inferred value. The difference is due to a large nonhydrostatic component from Tharsis and other sources. It may be that similar estimates can be made for Triton and Pluto without being too badly corrupted by other sources.

A spacecraft orbiting Triton and Pluto would give the best determinations of $J_{2}, C_{22}$, and $S_{22}$, as opposed to a flyby mission. A laser altimeter aboard an orbiter would also 
provide topographic and gravitational field data, just as in the case of Mars Global Surveyor (Zuber et al., 1998; Smith et al., 1999).

Hammel et al. (2001) propose a Neptune mission which might make repeated flybys of Triton. While not as useful as a Triton orbiter, such a mission would still provide valuable information about the satellite's gravity and topography.

A Pluto orbiter would be more involved. Unlike the proposed Neptune mission, which uses Neptune's atmosphere to slow down, aerobraking cannot be used at Pluto because its atmospheric envelope is too tenuous. The spacecraft's speed would have to be killed with rocket thrust, making the spacecraft heavier and more expensive.

\section{CONCLUSIONS}

Polar wander may occur on Triton due to the insolation-driven migration of volatiles. Triton's apparent large south polar cap might be caused by its wander toward the sub-Neptune point. This style of polar wander would imply that Triton's mantle has a relatively low viscosity. A low viscosity would likely agree with the leading side-trailing side cratering statistics and probably agree with the observed internal activity. Polar wander on Pluto due to volatile migration is more problematic, but still possible. Spacecraft measurements of Triton's gravitational field could give evidence for or against volatileinduced polar wander on that body. Spacecraft could possibly measure the degree of differentiation inside Triton and Pluto.

\section{Appendix}

This appendix gives a slightly more rigorous derivation of (24) via the power balance equation. The insolation and deposition of nitrogen at the surface will be assumed 
to be governed by the simple equation (e.g., Hansen and Paige, 1992; Spencer and Moore, 1992; Yelle et al., 1995):

$$
(1-A) F=\varepsilon_{s s} \sigma_{S B} T^{4}-L \frac{d \sigma}{d t}+k \frac{\partial T}{\partial z}
$$

where $T$ is Triton's surface temperature, $A$ is its albedo, $\varepsilon_{\mathrm{ss}}$ is its emissivity, and $k$ its thermal conductivity Also, $F$ is the insolation, $\sigma$ is the mass per unit area of solid nitrogen on the ground (measured in $\mathrm{kg} \mathrm{m}^{-2}$ ), $z$ is vertical direction (positive downwards), $t$ is time, while $L=$ $2.53 \times 10^{5} \mathrm{~J} \mathrm{~kg}^{-1}$ is the latent heat of sublimation for nitrogen and $\sigma_{\mathrm{SB}}=5.67 \times 10^{-8} \mathrm{Wm}^{-2} \mathrm{~K}^{-}$ ${ }^{4}$ is the Stefan-Boltzmann constant.

It will be assumed here that Triton's nitrogen atmosphere is always on the vapor pressure curve, and that solid nitrogen covers the satellite's surface completely; in this case $T$ is a constant across the satellite (Yelle et al., 1995). It will also be assumed that $A$ and $k$ are constant across the surface as well. The insolation $F$ and surface mass distribution $\sigma$ can be expressed in terms of spherical harmonics:

$$
\begin{gathered}
F=\sum_{\ell=0}^{\infty} \sum_{m=0}^{\ell}\left[F_{\ell m}^{C} P_{\ell m}(\sin \phi) \cos m \lambda+F_{\ell m}^{S} P_{\ell m}(\sin \phi) \sin m \lambda\right] \\
\sigma=\sum_{\ell=0}^{\infty} \sum_{m=0}^{\ell}\left[\sigma_{\ell m}^{C} P_{\ell m}(\sin \phi) \cos m \lambda+\sigma_{\ell m}^{S} P_{\ell m}(\sin \phi) \sin m \lambda\right]
\end{gathered}
$$

For the $\ell=0, m=0$ term, (A1) becomes

$$
(1-A) F_{00}^{\mathrm{c}}=\varepsilon_{s s} \sigma_{S B} T^{4}-L \frac{d \sigma_{00}^{\mathrm{c}}}{d t}+k \frac{\partial T}{\partial z}
$$


For all other terms, (A1) is

$$
\begin{gathered}
(1-A) F_{\ell \mathrm{m}}^{\mathrm{C}}=-L \frac{d \sigma_{\ell \mathrm{m}}^{\mathrm{C}}}{d t} \\
(1-A) F_{\ell \mathrm{m}}^{\mathrm{s}}=-L \frac{d \sigma_{\ell \mathrm{m}}^{\mathrm{s}}}{d t}
\end{gathered}
$$

Temperature drops out because $T$ is taken to be constant across the surface; it is therefore found only in the $\ell=0, m=0$ term given by (A4).

Only the $\ell=2, m=0$ term in (A5) affects $J_{2}$. In (A2) the insolation coefficient is

$$
F_{20}^{C}=F_{E}\left(\frac{a_{E}}{a_{N}}\right)^{2}\left(\frac{5}{16}\right)\left(\frac{3}{4} \sin ^{2} \varepsilon_{T}-\frac{1}{2}\right)
$$

(Rubincam, 1994). Thus integrating (A5) gives

$$
\sigma_{20}^{c}=\frac{5(1-A) F_{E}}{64 L}\left(\frac{a_{E}}{a_{N}}\right)^{2}\left\langle 3 \sin ^{2} \varepsilon_{T}-2\right\rangle t
$$

where by (2) and (3) in the main text

$$
<3 \sin ^{2} \varepsilon_{\mathrm{T}}-2>=\left(1-3 \cos ^{2} I \cos ^{2} \varepsilon_{\mathrm{N}}-3 \sin ^{2} I \sin ^{2} \varepsilon_{\mathrm{N}} / 2\right)
$$

The gravitational flattening due to the surface mass distribution can be shown to be (e.g., Kaula, 1968, p. 66): 


$$
J_{2, \text { vol }}=-C_{20, \text { vol }}=-2 \pi R_{\mathrm{T}}^{2}\left(\frac{2}{5}\right) \frac{\sigma_{20}^{\mathrm{C}}}{M_{\mathrm{T}}}=-\left(\frac{3}{5}\right) \frac{\sigma_{20}^{\mathrm{C}}}{\rho_{\mathrm{T}} R_{\mathrm{T}}},
$$

where $\rho_{\mathrm{T}}=2064.3 \mathrm{~kg} \mathrm{~m}^{-3}$ is the average density of Triton.

To overcome the rotational flattening

$$
J_{2, \text { rot }}+J_{2, \mathrm{vol}}=0 \text {. }
$$

so that the time $t_{\mathrm{S}}$ required for sublimation to overcome the rotational flattening will, by (A8)-(A10), be

$$
t_{\mathrm{S}}=\frac{64 L \rho_{\mathrm{T}} R_{\mathrm{T}} J_{2, \text { rot }}}{3(1-A) F_{\mathrm{E}}<2-3 \sin ^{2} \varepsilon_{\mathrm{T}}>}\left(\frac{a_{\mathrm{N}}}{a_{\mathrm{E}}}\right)^{2} .
$$

(A11)

This equation gives sublimation times almost identical to those given by (24) and (26).

\section{REFERENCES}

Bills, B. G., and T. S. James 1997. Polar motion of a viscoelastic Earth due to glacial cycle mass loading. J. Geophys. Res. 102, 7579-7602.

Brown, R. H., and R. L. Kirk 1994. Coupling of volatile transport and internal heat flow on Triton. J. Geophys. Res. 99, 165-1981. 
Croft, S. K., J. S. Kargel, R. L. Kirk, J. M. Moore, P. M. Schenk, and R. G. Strom 1995. The geology of Triton. In Neptune and Triton, (D. P. Cruikshank, Ed.), pp.879-947. Univ. of Arizona Press, Tucson.

Caputo, M. 1967. The Gravity Field of the Earth, Academic Press, New York.

Darwin, G. H. 1879. On the bodily tides of viscous and semi-viscous spheroids, and on the ocean tides upon a yielding nucleus. Philos. Trans. R. Soc. London 170, 1-35. (reproduced in Scientific Papers by G. H. Darwin, vol. 2, pp. 1-32, Cambridge University Press, New York, 1908.)

Dobrovolskis, A. R., and A. W. Harris 1983. The obliquity of Pluto. Icarus 55, 231-235.

Dobrovolskis, A. R., S. J. Peale, and A. W. Harris 1997. Dynamics of the Pluto-Charon binary. In Pluto and Charon (S. A. Stern and D. J. Tholen, Eds.), pp. 159-190. Univ. of Arizona Press, Tucson.

Folkner, W. M., C. F. Yoder, D. N. Yuan, E. M. Standish, and R. A Preston 1997. Interior structure and seasonal mass redistribution of Mars from radio tracking of Mars Pathfinder. Science 278, 1749-1752.

Goldreich, P., N. Murray, P. Y. Longaretti, and D. Banfield 1989. Neptune's story. Science, $245,500-504$.

Hammel, H. B., C. C. Porco, and K. Rages 2001. The case for a Neptune orbiter/multiprobe mission (abstract). In Forum on Innovative Approaches to Outer Planetary 
Exploration 2001-2020, p. 36. LPI Contribution no. 1084, Lunar and Planetary Institute, Houston.

Hansen, C. J., and D. A. Paige 1992. A thermal model for the seasonal nitrogen cycle on Triton. Icarus 99, 273-288.

Harris, A. W. 1984. Physical properties of Neptune and Triton inferred from the orbit of Triton. In Uranus and Neptune, (J. T. Bergstralh, Ed.), pp. 357-373, NASA Conference Publication 2330.

Jacobson, R. A. 1990. The orbits of the satellites of Neptune. Astron. Astrophys. 231, 241250.

Kaula, W. M. 1966. Theory of Satellite Geodesy, Blaisdell, Waltham, Mass.

Kaula, W. M. 1968. An Introduction to Planetary Physics, Wiley, New York.

Kirk, R. L., L. A. Soderblom, R. H. Brown, S. W. Kieffer, and J. S. Kargel 1995. Triton's plumes: discovery, characteristics, and models. In Neptune and Triton, (D. P. Cruikshank, Ed.), pp.949-989. Univ. of Arizona Press, Tucson.

Lambeck, K. 1980. The Earth's Variable Rotation, Cambridge Univ., Cambridge.

Lerch, F. J., S. M. Klosko, R. E. Laubscher, and C. A. Wagner 1979. Gravity model improvement using GEOS 3 (GEM 9 and 10). J. Geophys. Res. 84, 3897-3916. 
Marcialis, R. L. 1985. Topographic relaxation on ice-covered worlds: Application to Pluto (abstract). Bull. Am. Astron. Soc. 17, 715.

McKinnon, W. B., J. I. Lunine, and D. Banfield 1995. Origin and evolution of Triton. In Neptune and Triton (D. P. Cruikshank, Ed.), pp.807-877. Univ. of Arizona Press, Tucson.

McKinnon, W. B., D. P. Simonelli, and G. Schubert 1997. Composition, internal structure, and thermal evolution of Pluto and Charon. In Pluto and Charon (S. A. Stern and D. J. Tholen, Eds.), pp. 295-343. Univ. of Arizona Press, Tucson.

Rubincam, D. P. 1992. Mars secular obliquity change due to the seasonal polar caps. $J$. Geophys. Res. 97, 2629-2632.

Rubincam, D. P. 1994. Insolation in terms of Earth's orbital parameters. Theor. Appl. Climatol. 48, 195-202.

Rubincam, D. P. 2000. Pluto and Charon: a case of precession-orbit resonance? J. Geophys. Res. 105, 26, 745-26,755.

Smith, D. E., F. J. Lerch, R. S. Nerem, M. T. Zuber, G. B. Patel, S. K. Fricke, and F. G. Lemoine 1993. An improved gravity model for Mars: Goddard Mars Model 1. J. Geophys. Res. 98, 20,871-20,889.

Smith, D. E., M. T. Zuber, S. C. Solomon, R. J. Phillips, J. W. Head, J. B. Garvin, W. B. Banerdt, D. O. Muhleman, G. H. Pettengill, G. A. Neumann, F. G. Lemoine, J. B. Abshire, O. Aharonson, C. D. Brown, S. A. Hauck, A. B. Ivanov, P. J. McGovern, H. J. 
Zwally, and T. C. Duxbury 1999. The global topography of Mars and implications for surface evolution. Science 284, 1495-1503.

Spencer, J. R., and J. M. Moore 1992. The influence of thermal inertia on temperatures and frost stability on Triton, Icarus 99, 261-272.

Spencer, J. R., J. A. Stansberry, L. M. Trafton, E. F. Young, R. P. Binzel, and S. K. Croft 1997. Volatile transport, seasonal cycles, and atmospheric dynamics on Pluto. In Pluto and Charon (S. A. Stern and D. J. Tholen, Eds.), pp. 435-473. Univ. of Arizona Press, Tucson.

Stacey, F. D. 1992. Physics of the Earth, Third Edition, Brookfield Press, Brisbane, Australia.

Stern, S. A., and W. B. McKinnon 2000. Triton's surface age and impactor population revisited in light of Kuiper belt fluxes: evidence for small Kuiper belt objects and recent geological activity. Astron. J. 119, 945-952.

Ward, W. R. 1974. Climatic variations on Mars, 1. Astronomical theory of insolation. J. Geophys. Res. 79, 3375-3386.

Yelle, R. V., J. I. Lunine, J. B. Pollack, and R. H. Brown 1995. Lower atmospheric structure ans surface-atmosphere interactions on Triton. In Neptune and Triton (D. P. Cruikshank, Ed.), pp.1031-1105. Univ. of Arizona Press, Tucson.

Zuber, M. T., D. E. Smith, S. C. Solomon, J. B. Abshire, R. S. Afzal, O. Aharonson, K. Fishbaugh, P. G. Ford, H. V. Frey, J. G. Garvin, J. W. Head, A. Ivanov, C. L. Johnson, 
D. O. Muhleman, G. A. Neumann, G. Pettengill, R. J. Phillips, X. Sun, H. J. Zwally, W. Bruce Banerdt, and T. C. Duxbury 1998. Observations of the north polar region of Mars from the Mars Orbiter Laser Altimeter. Science 282, 2053-2060. 


\section{Table 1}

$\begin{array}{ccccccc}\lambda & J_{2, \text { rot }} & J_{2, \text { tides }} & J_{2, \text { hydro }} & C_{22, \text { ide }} & d & t_{\mathrm{S}} \\ \left(10^{-4}\right) & \left(10^{-4}\right) & \left(10^{-4}\right) & \left(10^{-4}\right) & (\mathrm{m}) & \left(10^{5} \mathrm{yr}\right)\end{array}$

Triton

$\begin{array}{lllllll}0.3 & 0.645 & 0.966 & 1.611 & 0.483 & 120 & 0.8\end{array}$

$\begin{array}{lllllll}0.4 & 1.325 & 1.986 & 3.311 & 0.993 & 250 & 1.6\end{array}$

\section{Pluto}

$\begin{array}{ccccccc}0.3 & 0.558 & 0.107 & 0.665 & 0.054 & 85 & 8 ? \\ 0.4 & 1.146 & 0.220 & 1.366 & 0.110 & 180 & 16 ?\end{array}$

Parameters for Triton and Pluto. $\lambda=C / M R^{2}$, where $C$ is the moment of inertia about the rotation axis, $M$ is mass, and $R$ is radius. $d$ is the equivalent depth of the global layer of solid $\mathrm{N}_{2}$ needed to make an ice cap which cancels the total hydrostatic flattening $J_{2}$, hydro on a rigid object, where $J_{2, \text { hydro }}=J_{2 \text {, rot }}+J_{2, \text { ide }} \cdot t_{\mathrm{S}}$ is the time needed to sublimate the equivalent global layer and condense it at the poles in order to overcome $J_{2, \text { hydro }}$. 


\section{Figure 1}

The Sun shining on the equatorial regions of Triton could cause volatiles (principally nitrogen) to migrate to the poles.

\section{Figure 2}

Path of polar wander on a rigid body. The ice caps alias the rotational flattening, leaving only the tidal bulge. The axis of wander is then the axis of the tidal bulge. Rotation about this axis would be expected to smear out leading side-trailing side cratering statistics.

\section{Figure 3}

Expected path of polar wander on body whose mantle has a low effective viscosity. The pole will wander so as to attempt to place the excess mass of the caps on the axis of the tidal bulge. The polar wander path is $90^{\circ}$ away from that shown in Figure 2, with the axis piercing the leading side and trailing side on the equator. Rotation about this axis does not disturb the leading side-trailing side cratering statistics. 


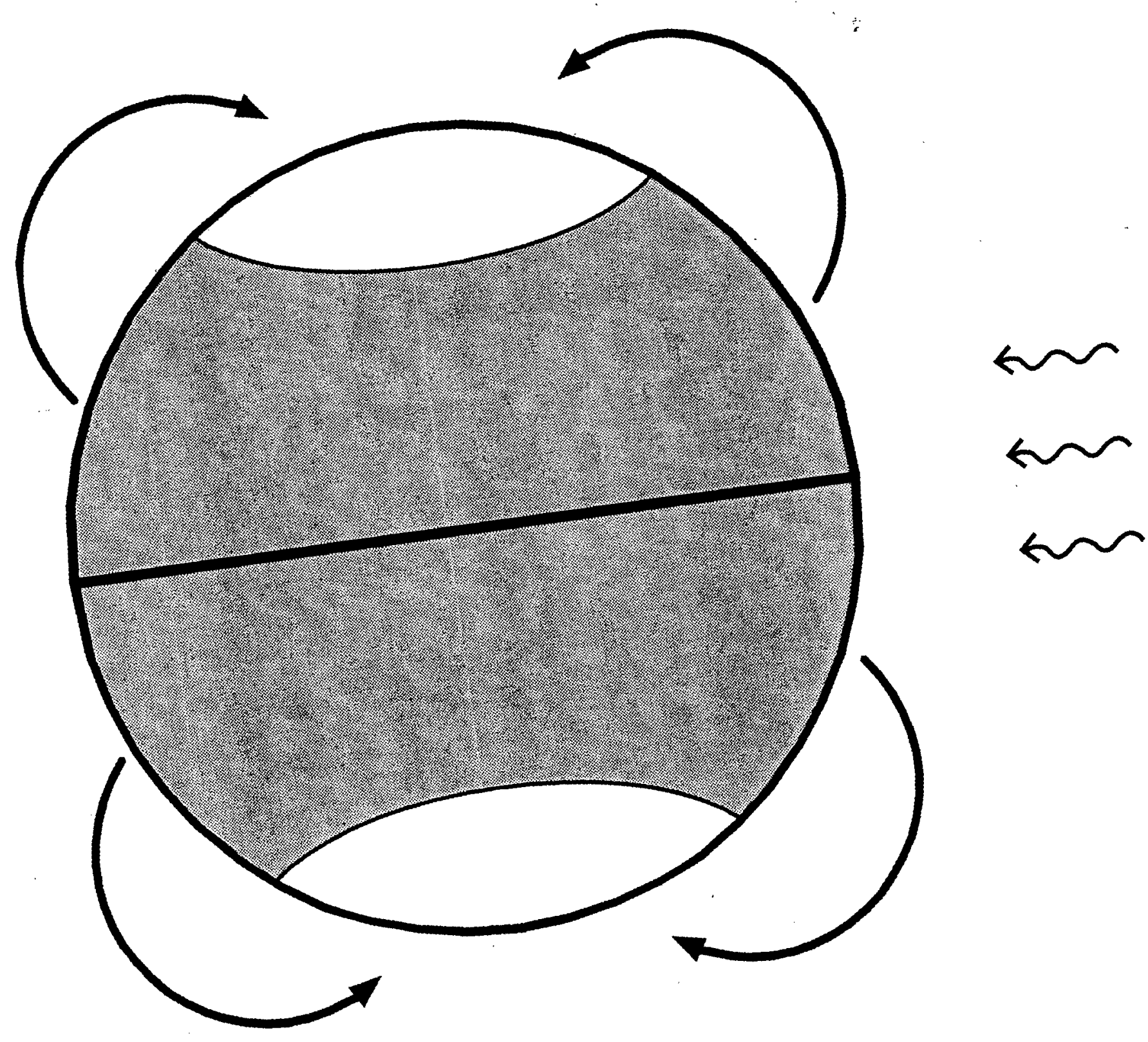

Figure 1 


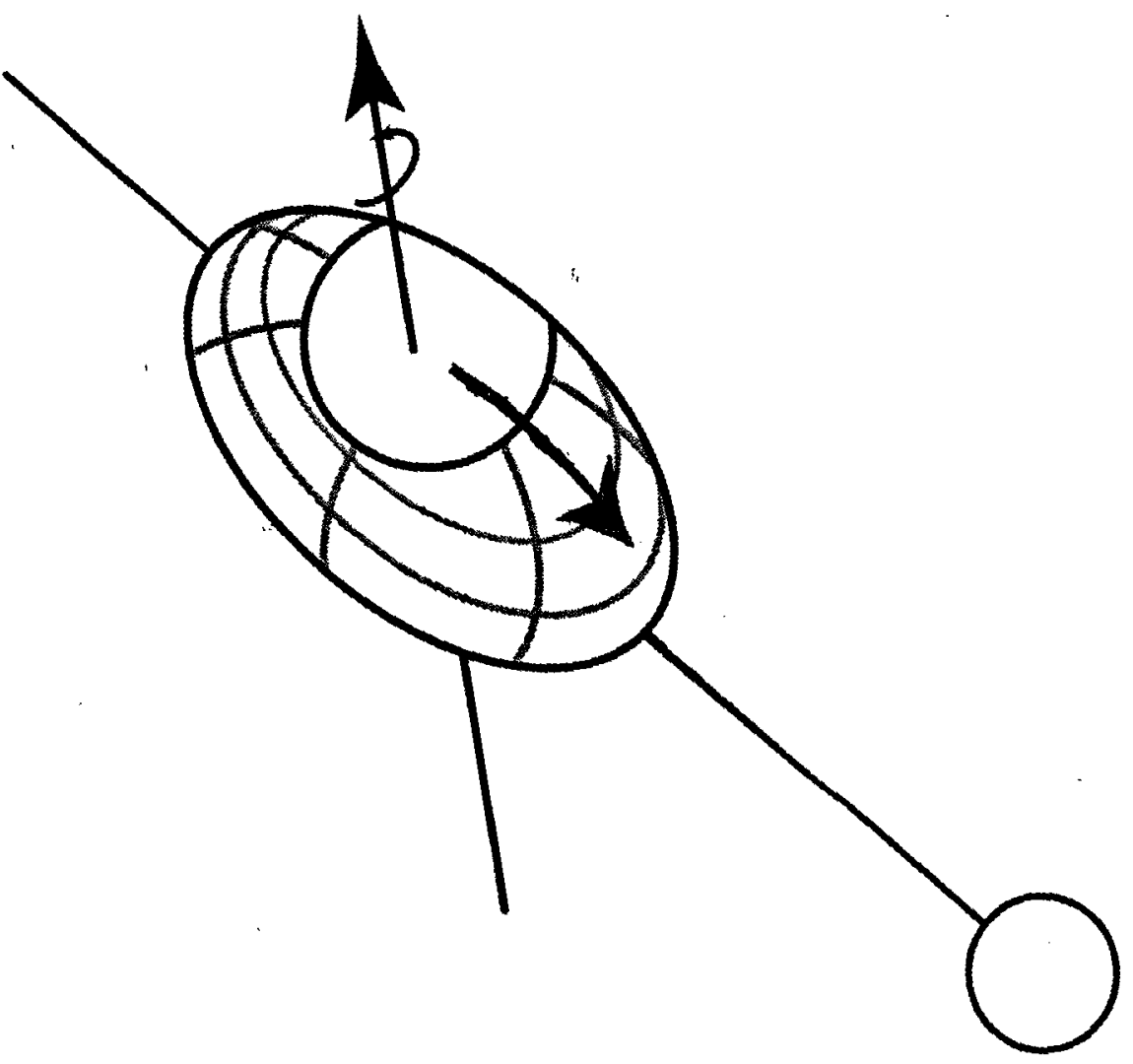

Figure 3 


\title{
Polar Wander on Triton and Pluto \\ Due to Volatile Migration
}

\author{
by \\ David Parry Rubincam
}

\section{POPULAR SUMMARY}

Polar wander may occur on Triton and Pluto because of volatile migration. Triton, with its low obliquity (obliquity is the tilt of the axis), can theoretically sublimate volatiles (mostly nitrogen) at the rate of $\sim 10^{14} \mathrm{~kg} \mathrm{yr}^{-1}$ from the equatorial regions and deposit them at the poles. Assuming Triton to be rigid on the sublimation timescale, after $\sim 10^{5}$ years the polar caps would become large enough to cancel the rotational flattening, with a total mass equivalent to a global layer $\sim 120-250 \mathrm{~m}$ in depth. At this point the moon overbalances and the pole wanders about the tidal bulge axis, which is the line joining Triton and Neptune. Rotation about the bulge axis might be expected to disturb the leading side/trailing side cratering statistics; the leading side bashes into more meteorites and asteroids. Because no such disturbance is observed, it may be that Triton is too stiff but its surface volatile inventory too low to permit wander. On the other hand, its stiffness might be low, so that any size cap might be expected to wander toward the tidal bulge axis. In this case, the axis of wander passes through the equator from the leading side to the trailing side; rotation about this wander axis would not disturb the cratering statistics. Polar wander may explain the bright southern hemisphere: this is the pole which is wandering toward the equator, while the other hemisphere tilts out of view. In any case the "permanent" polar caps may be geologically very young. Polar wander may also take place on Pluto, due to its obliquity 
oscillations and perihelion-pole geometry. However, Pluto is probably not experiencing any wander at present. The Sun has been shining strongly on the poles over the last million years, so that volatiles migrate to the equator, stabilizing the planet against wander. Spacecraft missions to Triton and Pluto which measure how flat the objects are could give information about the accumulation of volatiles at the poles. Such information is best obtained by measuring gravity and topography from orbiters, as was done for Mars with the highly successful Mars Global Surveyor. 